\title{
Las instituciones de educación superior y su rol en la era digital. La transformación digital de la universidad: ¿transformadas o transformadoras?

\author{
Higher education institutions and their role in the Digital Age. \\ The digital transformation of the university: transformed or transforming?
}

Ernesto Chinkes ${ }^{1}$ ORCID 0000-0001-6092-537X

David Julien² ORCID 0000-0003-1820-7483

Recibido: $1 / 3 / 19 \cdot$ Aprobado: 21/4/19

Cómo citar: Chinkes, E., \& Julien, D. (2019). Las instituciones de educación superior y su rol en la era digital. La transformación digital de la universidad: ¿̇transformadas o transformadoras? Ciencia y Educación, 3(1), 21-33. Doi: https://doi.org/10.22206/cyed.2019.v3i1.pp21-33

\section{Resumen}

Este artículo nos introduce en la era digital, tratando de evidenciar la relevancia e impacto que tiene en la sociedad contemporánea. Se identifican las principales tecnologías disruptivas que la están formateando y cómo, a partir de estas, se están transformando los modelos y procesos de las organizaciones, generando cambios profundos, abruptos y a la vez efímeros. Desde dicho lugar se analizan los desafíos de las universidades en esta era, sus riesgos, oportunidades y lineamientos para encarar la transformación digital de las mismas. Esta transformación se plantea como una necesidad que debe ser abordada sin dilaciones, pero con visión crítica y bajo las particularidades de cada institución. También se intentan esbozar algunas ideas sobre el rol con el que las instituciones de educación superior podrían contribuir para construir una sociedad del conocimiento más saludable, y cuál es la estrategia que está proponiendo la Organización Universitaria Interamericana (OUI) para actuar como facilitadora de este cambio.

Palabras clave: era digital; transformación digital; universidad; tecnologías disruptivas.

\begin{abstract}
This article introduces us to the digital age, presenting evidence of the relevance and impact that it has in today's society. We identify the main disruptive technologies that are formatting it and how is it that the models and processes of the organizations are being transformed generating deep, abrupt and at the same time ephemeral changes. From this assertion we analyze the challenges, risks, opportunities and guidelines that universities must face to address their own digital transformation. This transformation is understood to be inevitable and that it must be addressed without delay but with a critical vision and a clear understanding of the particularities of each institution. It also outlines some ideas about the role that higher education institutions could play to make contributions in building a healthier knowledge society, and what is the strategy that the Inter-American Organization for Higher Education (OUI-IOHE) is putting forth to act as facilitator of this change.
\end{abstract}

Keywords: Digital era; digital transformation; university; disruptive technologies.

1. Consultor Internacional, Asesor en Transformación digital, Organización Universitaria Interamericana (OUI), Canadá

Correo-e: echinkes@gmail.com

2. Secretario General Ejecutivo de la Organización Universitaria Interamericana (OUI), Canadá

Correo-e: david.julien@oui-iohe.org 
Las instituciones de educación superior y su rol en la era digital. La transformación digital de la universidad: ¿transformadas o transformadoras?

\section{Introducción}

\section{La era digital}

Estamos transitando un momento de la historia en donde las tecnologías de la información y los datos que generan, procesan, almacenan y transmiten son los grandes protagonistas. Su incorporación masiva viene promoviendo cambios profundos y rápidos en la sociedad; en sus formas de trabajo, de relacionarse, de divertirse, de producir, de consumir, de aprender y de generar el conocimiento. Se producen, por lo tanto, nuevas dinámicas sociales, políticas, culturales, económicas y de apropiación del conocimiento.

Hay quienes hablan de la cuarta revolución industrial (Schwab, 2016), aunque aquí preferiremos referenciarlo como un cambio de "era" (hacia la era digital). En esta el predominio sería de los servicios y la denominada "experiencia del usuario" por sobre la producción de bienes. Servicios impulsados por la digitalización y las tecnologías relacionadas. Se incorpora "inteligencia" en los productos y se les adicionan servicios digitales, se rediseñan procesos en forma innovadora (pudiendo automatizarlos, agilizarlos, optimizarlos y personalizarlos) y convierte, en muchos casos, la provisión de bienes en la prestación de servicios. La economía digital también potencia el concepto de internacionalización, ya que más que nunca las fronteras se hacen difusas y se minimizan las limitaciones para una sociedad global y de la inmediatez.

Este momento representa una gran amenaza para quienes no estén preparados, no entiendan o no tengan la capacidad de transformarse y adaptarse. Pero también, como una enorme oportunidad para generar valor, bienestar, prosperidad y empleos de calidad. América Latina y el Caribe pueden focalizar su energía para trabajar en forma coordinada y sinérgica, y aprovechar las oportunidades que este cambio de época le otorgará a los que se muevan más rápida e inteligentemente. Las universidades, así como el resto de los miembros del sistema educativo y científico, pueden jugar un papel central en el ecosistema digital que dominará este siglo.

Este artículo hace un recorrido por algunas de las tecnologías que motorizan estos cambios, describe su impacto en modelos, procesos, y la transformación digital de las organizaciones. En la cuarta sección se aborda el foco central del artículo, que es la transformación digital de las instituciones de educación superior, aportando lineamientos, riesgos, oportunidades y alertas. En la quinta, se intenta generar un aporte a la sociedad del conocimiento, planteando reflexiones sobre el rol que pueden jugar las universidades en la construcción de esta nueva era. Por último, en la sección final, la Organización Universitaria Interamericana (OUI) presenta las líneas de acción que está proponiendo para avanzar en este camino.

\section{Un recorrido por las tecnologías}

Hay una serie de tecnologías que destacan por sus habilidades para apalancar la disrupción de procesos y modelos que identifican esta época. Algunas de ellas podrían referenciarse como tecnologías de infraestructura de base; otras, como soluciones tecnológicas más específicas, que son posibles gracias a las primeras.

Los grandes avances de las tecnologías de base se vislumbran en el aumento exponencial en las capacidades de procesamiento, conectividad y almacenamiento, y en una drástica disminución de sus costos, que posibilitaron en muchos casos su masificación. Estos avances se vieron potenciados además con la aparición de la "nube", que facilitó el acceso a estas capacidades tecnológicas sin necesidad de grandes inversiones de capital (usándolas bajo demanda). Estas mayores capacidades de la infraestructura de base permitieron el surgimiento de conceptos y soluciones tecnológicas como big data, analytic, inteligencia artificial, impresión 3D, realidad aumentada, teléfonos inteligentes de última generación y apps avanzadas, sensores de todo tipo, IoT (Internet of Things), blockchain, etc. Estas tecnologías digitales se potencian entre sí, también con otras tecnologías del mundo físico, por ejemplo, los avances en la ingeniería de materiales, la nanotecnología, la robótica y la biotecnología.

El "Big Data" caracteriza la existencia de grandes volúmenes de datos sobre muchas de las personas, 
objetos y eventos que nos rodean, evidenciando un crecimiento en forma exponencial; generando desafíos tecnológicos para su gestión y, a su vez, oportunidades para aprovecharlos por parte de las instituciones (Chinkes et al., 2015). Existen datos tanto internos como externos a las instituciones, datos estructurados, semiestructurados y no estructurados (como los mensajes, textos, audios y videos) y la posibilidad de usar nuevas y diversas fuentes, como las redes sociales, los datos que generan los teléfonos inteligentes, equipamiento de todo tipo conectado a Internet (Chandler, 2015, p. 835). Por su parte, se denomina "Analytic" a la capacidad de aprovechar esos datos para generar valor, brindando información para la toma de decisiones o al incorporar algoritmos denominados "inteligentes" (basados en datos) en productos, servicios y procesos.

Relacionados con estos conceptos se ubica la inteligencia artificial, que busca imitar y potenciar las capacidades de los seres humanos para analizar, diagnosticar, buscar y encontrar ideas, aprender del pasado, razonar e interactuar de un modo natural con otras personas. Gran parte de esa "inteligencia" se genera mediante algoritmos que originan modelos basados en los datos (tecnologías bajo el rótulo de machine learning $y$ deep learning). La inteligencia artificial viene avanzando a pasos agigantados en los últimos años y se espera aún mucho más para los que siguen (Bostrom, 2014).

La Internet de las cosas (IoT - Internet of Things) refiere a los objetos conectados en red, que puedan capturar, enviar y recibir datos, así como incorporarles cierta "inteligencia” (que también puede ser transmitida por Internet). De esta forma los objetos tienen la posibilidad de capturar distinto tipo de mediciones de la realidad del objeto y su entorno, y luego enviar todos esos datos; permitiéndoles luego recibir instrucciones de cómo es la mejor forma de cumplir su función.

Las impresoras 3D, a su vez, están logrando lo que parecía imposible. El mundo digital siempre estuvo limitado a las lógicas de circulación de la información. Mientras que el flujo de los datos evolucionaba a pasos gigantes, el mundo físico se convertía en un gran contrapeso que limitaba sus posibilidades.
Una venta en línea, que podía realizarse al otro lado del continente en milisegundos, luego debía tardar varios días para trasladar el bien. Las impresoras 3D están desdibujando esos límites. El software permite personalizar el diseño del producto según las necesidades del usuario, luego ese diseńo puede viajar de manera digital y, finalmente, la producción del bien es su "impresión" que se puede hacer en tiempo real y cerca del consumidor final, minimizando costos de mano de obra y logística. Cuanto más se abaraten las impresoras y más fácil sea el uso del software, más cerca estará el consumidor final de eliminar los límites entre ambos mundos. En la actualidad, las impresoras 3D permiten la fabricación de bienes en diversos materiales, ya sean repuestos de todo tipo, así como de comidas, calzados, prótesis, casas, etc. (3Dnative, 2018).

La tecnología "blockchain” está posibilitando nuevos esquemas para concretar acuerdos entre pares, sin intermediarios, con mayor seguridad, rapidez y transparencia. Este tipo de soluciones tecnológicas abre la posibilidad de generar contratos inteligentes que se ejecuten automáticamente, o certificaciones que aseguren mayor certeza a los involucrados (Grech \& Camilleri, 2017). Se plantean, por lo tanto, soluciones por industria que posibilitan lógicas de interacción más ágiles, pero a la vez más seguras y con menores costos.

La tecnología de la "realidad aumentada" permite enriquecer las percepciones de la realidad que captamos a través de nuestros sentidos, complementándolas con datos que incorpora el mundo digital. Superpone en tiempo real imágenes, sonidos, marcadores y otro tipo de información sobre los elementos físicos percibidos. Si bien existen lentes especiales de esta tecnología, están surgiendo aplicaciones que aprovechan las capacidades de los teléfonos inteligentes y que seguramente lograrán masificar su uso.

Los dispositivos móviles, y en particular los teléfonos inteligentes, no han dejado de evolucionar y masificarse en la última década. Han permitido que muchas personas tengan en su bolsillo acceso a un potente equipo de cómputo multipropósito en todo momento y lugar. Existen millones de aplicaciones disponibles que extienden la potencialidad de estos dispositivos, otorgándoles el máximo aprovechamiento 
de sus capacidades de procesamiento, almacenamiento y conectividad, en sus cámaras, micrófonos, parlantes, GPS, sensores de movimiento, de huellas dactilares, etc. Por otro lado, hay una comunidad creativa muy enérgica que desarrolla y comparte aplicaciones, haciendo uso de las tecnologías descritas para múltiples propósitos y explotando sus características al máximo.

Lo interesante es que estas tecnologías se potencian entre sí, acelerando su apropiación masiva, el impacto y el alcance de las soluciones que se construyen; pero también acortando los tiempos en los que surgen nuevas soluciones, ya que la tecnología también se usa para construir nueva tecnología en forma más rápida y fácil.

\section{Los nuevos modelos y la transformación digital}

La invención de soluciones, basadas en las tecnologías descritas, generan nuevos modelos de negocio y dinámicas organizacionales y sociales, que en muchos casos hacen desaparecer a las instituciones que se basaban en los modelos preexistentes. Se puede evidenciar esta realidad cuando se analiza el surgimiento de una empresa como Netflix, que creó una plataforma para ofrecer un servicio de películas bajo demanda y tuvo un impacto devastador en las empresas que proveían el servicio de alquiler de películas (inclusive para Blockbuster, una empresa multinacional muy poderosa), puesto que implicaba ir a buscar DVDs y luego devolverlos a las 24 horas. O lo sucedido con el negocio de los rollos de fotografía y su revelado, que fueron desplazados por las cámaras digitales y luego estas bajaron enormemente su demanda por la gran capacidad de los teléfonos inteligentes para cumplir esta función. También una empresa que fabricaba y comercializaba dispositivos GPS (como Garmin ${ }^{\circ}$ ) dejó obsoletas a las que hacían y comercializaban guías y mapas, pero luego estas fueron reemplazadas por aplicaciones que se instalan en los teléfonos inteligentes (como Google Maps o Waze) que no tienen costo adicional y permiten una masificación mucho mayor; además suman funcionalidades en tiempo real.

Las plataformas de transporte de pasajeros (como Uber), de envíos (como Rappi y Glovo), o de hospedaje (como Airbnb) permiten conectar individuos y empresas que ofrecen un servicio con otros que desean consumirlo; hacen repensar negocios y sectores que habían funcionado de otra forma durante años. Las agencias de viajes virtuales, consultas médicas o legales en forma virtual (incluso mediante bots) y las impresiones 3D, que permiten eliminar los costosos stocks de piezas destinadas al mantenimiento o la realización de prótesis a medida del paciente y que se realizan en el momento con solo "imprimirlas", son otros ejemplos de las disrupciones en los modelos tradicionales que están sucediendo.

Todos ellos demuestran cómo pueden mejorarse los productos, servicios y canales para provisionarlos; eliminar intermediarios y repensar procesos operativos que permitan disminuir costos y aumentar rapidez. Es interesante observar, también, que su éxito puede ser efímero. Un estudio muestra que el $52 \%$ de las empresas que estaban en la lista Fortune 500 en el año 2000 , a fines de 2014 dejaron de estarlo; habían quebrado, fueron compradas, desaparecieron o bajaron de la lista (Hildebrand, 2014). Todo lleva a pensar que en la actualidad se han acelerado estos cambios, e intuir que en el futuro puede ser mayor aún.

La transformación digital de una organización requiere incorporar tecnologías, crear o modificar procesos y disponer de las personas con las capacidades y habilidades adecuadas para dichos procesos y tecnologías. Es por ello que los mayores progresos han ocurrido en compañías que son nativas digitales, en contraste con las organizaciones más maduras. Modificar procesos, personas y tecnologías es muy difícil cuando están muy consolidadas en su cultura organizacional. Por otro lado, ser digital no asegura el éxito organizacional, hay varias empresas que han puesto mucha energía y dinero en transformarse digitalmente y han fracasado, puesto que ello no implica, necesariamente, que el mercado acepte el producto, lograr canales y procesos de producción y comercialización que lo hagan rentable o generar las ganancias con la velocidad que le exigían sus accionistas (Davenport \& Westerman, 2018). Por lo tanto, podríamos concluir que ser digital no es un objetivo, sino un medio para lograr los objetivos; y que no es 
una tarea sencilla, ya que implica mucho más que incorporar tecnología.

\section{La transformación digital y las instituciones de educación superior}

Lo descrito expone una revolución que, basada en los datos digitales y las tecnologías asociadas, ubica al "conocimiento" en el centro de la escena social, económica y política. Pero teniendo en cuenta que el conocimiento es el área de incumbencia de las instituciones de educación superior, cabe interrogarse cuál es el rol que estas deben desempeñar en esta nueva era. ¿Es un papel destacado, o, por el contrario, la participación de nuevos actores en la generación y distribución del conocimiento otorga a las universidades una menor relevancia que la que tenían en otros tiempos?

Por un lado pareciera que es un gran momento para estas instituciones, la era digital está poniendo en primer plano, y con un nivel de relevancia nunca visto, al elemento que las universidades dominan y vienen manejando desde hace siglos: "el conocimiento"; sin embargo, esto contrasta con otra visión, con fundamentos válidos, donde la importancia de las universidades empieza a disminuir, ya que hay ahora otros ámbitos donde las personas pueden obtener los conocimientos. Esta contradicción aparente denota que es un escenario complejo, que necesita un análisis más cuidadoso y detallado.

En primer lugar, se puede observar que hoy existe un ecosistema de generación y distribución del conocimiento por fuera de las universidades. Está basado en Internet y conformado por diversas plataformas de contenidos y de colaboración que se establecen como ámbitos válidos de gestión del conocimiento, en donde participan personas e instituciones de diversa índole.

Son ejemplos las plataformas de videos en línea (como Youtube) donde existen una gran cantidad de videos que enseñan temáticas sobre diversas disciplinas, y que son usados por millones de personas diariamente para aprender. También las plataformas de cursos masivos en línea, abiertos, denominados
MOOCs y que habilitan la enseñanza de programas completos sobre determinadas temáticas; están debidamente estructurados, con videos, documentos, autoevaluaciones y demás recursos para cualquiera que desee acceder desde algún lugar del planeta con conexión a Internet, incluso, en muchos casos, de manera gratuita. En este mismo orden de ideas, las enciclopedias abiertas como Wikipedia o los buscadores como Google ofrecen rapidez y fácil acceso a libros, a publicaciones científicas y de divulgación digitales en todo el mundo sin tener que moverse del lugar donde se encuentre un individuo. Los repositorios abiertos, que muchas instituciones alrededor del planeta están propiciando, acrecientan más este recurso, ya que, a diferencia de muchas revistas científicas, permiten acceder a los textos completos sin necesidad de pago alguno.

También existen portales y blogs donde personas entusiastas, y no por ello menos formadas, divulgan sus conocimientos; o los foros, donde hay individuos que interrogan y otros que contestan sobre las más variadas áreas del conocimiento. Todos ellos tienen a la mano alternativas para comunicarse en forma directa, rápida y ágil; tanto los que quieren aprender sobre un tema como los que quieren enseñarlo, ya sea por correo electrónico, mensajería instantánea o asincrónica, o video conferencia, solamente disponiendo de un equipo conectado a Internet.

Se suman a este ecosistema empresas e instituciones, como editoriales o plataformas, que incursionan en la enseñanza, debido a que encuentran sinergias potentes. Es el caso de LinkedIn (comprada por Microsoft en 2016, y que posee la base de datos de candidatos con sus respectivos currículum más grande del mundo), que permite conocer mejor que nadie las necesidades del mercado laboral y su capacidades, y que está incursionando en propuestas como LinkedIn Learning, plataforma de formación (un MOOC) que puede aprovechar todo el conocimiento de sus datos y algoritmos para personalizar los cursos a las necesidades del mercado laboral (Ariño, 2018, p. 11).

Las posibilidades de acceso al conocimiento, en la actualidad, no quedan restringidas a los ámbitos locales donde el individuo habita, sino que su alcance se amplifica al mundo gracias a Internet. Son miles 
de millones de individuos. En 2018 son 3.8 miles de millones los que se encuentran conectados a Internet (Data Never Sleeps 6 | Domo, 2018). El conocimiento difundido en formato digital no tiene fronteras geográficas y empiezan a desaparecer también las del lenguaje con las posibilidades de traducción en línea, que es cada vez de más fácil uso y de mejor calidad.

Estos elementos, relativos a la generación y apropiación del conocimiento, conforman un escenario totalmente distinto al que estuvieron acostumbradas las universidades durante siglos. En este contexto, pareciera que una institución de educación superior que se limite a sus capacidades endógenas y que además no cumpla con las exigencias de experiencia de usuario a los que hoy está acostumbrada la sociedad (ubicuidad, 7x24, personalización, etc.), deberá enfrentar una batalla muy desigual. Son millones de personas distribuidas por el mundo que disponen de herramientas de fácil acceso, que periódicamente aumentan sus capacidades individuales para encontrar, generar y distribuir conocimiento y que contrastan con las de unos pocos miles que lo pueden hacer dentro de cada universidad. Por otro lado, en el ecosistema planteado no son solo individuos aislados, también son empresas que se benefician del trabajo de estos y que les facilitan plataformas para que puedan hacerlo mejor, o empresas que detectan en la generación y difusión del conocimiento un negocio rentable y sinérgico con otros.

Aquellas universidades que no perciban estos cambios y consideren que pueden seguir haciendo lo que hacían (aunque era totalmente válido hace tan solo unos años atrás) posiblemente tendrán dificultades en el futuro cercano para encontrar su rol en esta sociedad. Por otro lado, existen en la actualidad posibilidades inéditas para la generación, gestión y distribución del conocimiento y las universidades tienen la oportunidad de potenciar al máximo su misión de enseñanza, investigación y vinculación con su entorno.

Entonces, la contradicción que se evidenciaba a primera vista no era tal. Algunas universidades podrán brillar más que nunca, otras serán tan opacas que será difícil distinguir su utilidad en esta era; dependerá posiblemente de su capacidad de adaptación. Los alumnos, docentes e investigadores de las universidades son grupos intelectualmente selectos, muy formados, comprometidos con el conocimiento, que dedican gran tiempo y energía a ello; en estos tiempos tienen la posibilidad de trabajar en forma conectada, colaborativa y sinérgica.

Pero muchas de estas posibilidades que hoy brinda esta era las tiene cada individuo a su disposición sin la universidad como mediadora. El desafío que tienen las instituciones académicas es reflexionar con espíritu crítico sobre su rol, para poder "repensarse" en esta época. De este análisis seguramente detectaremos que la universidad no debiera estar limitada por días, horarios, ni al acceso presencial para todas sus actividades; debe aprovechar mejor los datos que tienen a su disposición (entender mejor las necesidades del mercado laboral, las necesidades de la sociedad, y las de sus estudiantes, docentes e investigadores). Son los elementos que se exigen en la actualidad para todos los servicios que desean subsistir. También debe generar una cultura de la colaboración. Por último, ser más flexibles y ágiles con mejores capacidades de adaptación.

Podemos mencionar dos grandes retos estratégicos que las universidades de América Latina y el Caribe debieran considerar:

\section{Reto 1: aprovechar mejor las sinergias entre los miembros de la comunidad universitaria}

Es necesario explorar de qué forma la institución puede impulsar el trabajo de los individuos que la integran, y lograr que el todo (la universidad) sea más que la suma de sus integrantes (gracias a la sinergia y potencia de sus interrelaciones). La universidad tiene un potencial enorme con relación al conocimiento y debe generar dinámicas que, a través de las tecnologías, aprovechen la colaboración entre los actores de la propia institución, pero sin que pierdan las riquezas que también existen fuera de ellas.

Muchas veces las áreas de las universidades funcionan como "silos", donde los distintos departamentos, centros, institutos, cátedras, etc. trabajan y funcionan de manera aislada del resto. Las tecnologías de la 
información y la comunicación son grandes habilitadores para apoyar las conexiones que son necesarias. Deben integrarse bajo dos ejes: 1) enseñanza, investigación y extensión; y 2) las múltiples áreas disciplinares (particionadas en facultades, departamentos, centros, materias, cátedras, etc.). Todo ello debe trabajar más conectado y aprovechando también las redes de apoyo externas de cada uno de sus referentes. Las tecnologías pueden generar repositorios de conocimiento compartido, habilitar plataformas que posibiliten el armado de grupos de trabajo colaborativo y también faciliten redes de contactos y de apoyo que identifique especialistas en las distintas disciplinas, favorecer su comunicación, etc. Pero todo esto puede funcionar si, además de ofrecer plataformas tecnológicas que lo soporten, se generan políticas que motiven y promuevan estas dinámicas, y permitan modificar una cultura organizacional muy consolidada a lo largo de los años.

Lo anterior es uno de los aspectos fundamentales para generar lo que podemos llamar la "universidad inteligente". Implicaría lograr que las capacidades de una institución sean mayores que la de la suma de sus miembros, ya que se potencian las capacidades de cada uno de ellos cuando participan dentro de la universidad. Es decir, donde cada uno puede dar mucho más perteneciendo a la universidad que si lo hiciera solo. Ello se logra con auxilio de la tecnología, pero implica, principalmente, cambios culturales fuertes.

\section{Reto 2: abandonar la lógica "monopólica” que obligue a interrogarse y superarse periódicamente}

Las instituciones necesitan autoevaluarse con periodicidad y compararse con el entorno, para cuestionarse si no lo podrían hacer mejor.

Empecemos, por ejemplo, con el objetivo institucional de la enseñanza. La universidad debe comprender por qué un individuo elegiría aprender en ella. Implica salirse del esquema de comodidad que, haciendo la comparación con el mundo empresarial, se da en las corporaciones que son "monopólicas" y que hacen pocos esfuerzos por mejorar sus productos y servicios, ya que tienen a sus clientes cautivos.

Esta incomodidad, que se incrementará con el paso de los ańos, debe motivar a las instituciones para hacerlo cada vez mejor y para repensar su existencia. Es cuestionar y definir qué es lo que están haciendo para que un estudiante dentro de su universidad aprenda más y mejor que el que lo haga por fuera de ella. Debe compararse no solo con otras universidades cercanas, sino con otras muy distantes y con muchos otros actores que no son universidades.

Muchas de las tecnologías descritas habilitan a las universidades para generar ambientes de aprendizaje más ricos, que mejoran la forma de enseñar y de aprender. La realidad virtual, la realidad aumentada y las simulaciones pueden apoyar la experimentación y aplicación de conocimientos que no serían posibles de manera tradicional, o si lo fuesen, deberían restringirse a muy pocos casos. Esto es, la posibilidad de que un estudiante novato de medicina, odontología o veterinaria realice una cirugía y que el paciente no sufra consecuencias negativas de esta práctica; o un estudiante de ingeniería haga los cálculos para construir un puente y luego ver si resiste el paso de un camión, sin arriesgarse a que suceda una catástrofe ¿̨Se imagina si un estudiante de historia o antropología pudiera vivenciar momentos de la historia con todos sus sentidos, y analizarlo por múltiples dimensiones, $\mathrm{y}$ visiones?

Las posibilidades que hoy existen para que los alumnos y docentes aprendan mediante videos, con un diseño didáctico, que faciliten la asimilación de los conceptos y que, a su vez, puedan repetirlo las veces que sea necesario desde cualquier lugar y momento, los convierte en una potente herramienta formativa. Adicionalmente, gracias a las facilidades de edición que existen es posible su generación en forma autónoma por distintos actores de la comunidad universitaria como investigadores, docentes, alumnos, e inclusive, egresados. Las universidades pueden además agregarle la certificación institucional, para que a esos materiales educativos se los distinga de cualquier otro que circule por Internet. Posibilita entonces habilitar estrategias pedagógicas muy potentes que deben ser aprovechadas en forma integral dentro de las universidades.

De igual forma las plataformas colaborativas (mensajería, conferencias de voz o de video, compartición o escritura de documentos grupales, etc.) facilitan la construcción de conocimiento en forma colectiva, así como ofrece nuevas posibilidades de comunicación entre 
estudiantes y/o docentes, ampliando los límites que impone el aula física y la conversación cara a cara.

Las universidades deben generar dinámicas de aprendizaje en las que el aprovechamiento de estas herramientas ocurra de forma colaborativa, de manera que sean más potentes que cuando las usen los individuos en forma aislada. Por ejemplo, redes de apoyo con las personas y en los momentos de aprendizaje adecuados, a través de nuevas dinámicas de interacción que superen al espacio físico sin restricciones de tiempo y locación, con docentes y estudiantes de materias relacionadas de otras o la propia institución y con investigadores de distintas áreas u organizaciones. También deben considerarlas para pensar actividades que permitan formar al estudiante con visiones de miembros de otras culturas y países (con docentes y estudiantes de otras regiones), incorporando en la práctica habitual nociones tan importantes como la internacionalización en la enseńanza.

Esta era digital también permite aprovechar los datos que existen en las instituciones y fuera de ellas. Posibilita, entonces, personalizar aprendizajes y detectar problemas o alertas tempranas, mejorar su ámbito de estudio, optimizar servicios, etc. También pueden aprovechar los dispositivos móviles (teléfonos, tabletas, etc.) que llevan los alumnos y docentes (en todo momento y lugar) para desarrollar actividades didácticas, o generar software interactivo para la enseñanza (apps). Las oportunidades que hay en la actualidad son enormes, y muchas más las que surgirán en el futuro, por eso lo fundamental es que exista una institución con las capacidades de evaluar periódicamente cómo aprovecharlas y que disponga de la flexibilidad para implementar las más adecuadas que logren impactar en los aprendizajes.

De igual manera pasa con la ciencia y la forma de investigar, o en la extensión universitaria. Se ven impactadas por la disrupción que genera la era digital. También ameritan una interrogación profunda para analizar si no se podría hacer mejor, aprovechando todos los recursos disponibles de esta época y generando las transformaciones internas para aumentar sus capacidades.

Es tan fuerte el impacto en la ciencia, que existen actualmente diversos enfoques epistemológicos donde se pone en discusión si se deben usar los datos para testear las teorías o si directamente los datos y los algoritmos analíticos son los que descubren el conocimiento (Kitchin, 2014). Pero no es objeto de este texto profundizar esta discusión, ni sacar conclusiones o establecer posturas; solo evidenciar que la investigación científica claramente está fuertemente revolucionada por la era digital y las posibilidades que abre son enormes.

Las tecnologías existentes permiten medir muchos acontecimientos que antes eran imposibles. Los sensores amplían en cantidad y calidad las capacidades sensoriales de los seres humanos. Por otro lado, las ciencias sociales pueden obtener patrones del comportamiento humano como nunca antes en su historia, ya sea por el uso de las redes sociales o por otras tecnologías de uso masivo que ayudan a medir sus hábitos y acciones. Hay posibilidades de colaboración en tiempo real con otros investigadores, sin importar en qué lugar del planeta se encuentren, o de hacer un uso compartido de instrumentos avanzados, de compartir datos entre grupos de investigación distribuidos, de realizar trabajos complementarios, etc. Las instituciones que más recursos habiliten a sus investigadores para que aprovechen al máximo estas capacidades estarán multiplicando exponencialmente las posibilidades para el descubrimiento. Deben proveerles acceso a tecnología de punta que solo esté disponible en las universidades y facilitarles ambientes de trabajo colaborativo, desmotivando el trabajo en "silos". Por último, las capacidades de generación, almacenamiento y uso de grandes volúmenes de datos desdibujan el concepto de muestra (y se intenta trabajar en forma más cercana a la población total). Por lo tanto, proveerles grandes capacidades de almacenamiento es también una prioridad.

La vinculación de las universidades con su entorno es otro objetivo que también puede ser repensado y resignificado en esta era y que se profundiza en la siguiente sección.

La universidad, gracias a las tecnologías, puede acercarse a la población en forma masiva para brindarle conocimientos. A diferencia de otros actores, puede ofrecer niveles de certificación y de confianza, que en tiempos de la posverdad es indispensable. Por 
otro lado, se habilitan mecanismos para recibir datos del entorno (mediante el uso de apps o sensores en diversos ámbitos), o la validación de hipótesis sobre el impacto de teorías sociales. La posibilidad única de captar las necesidades sociales, la de rectificar o refutar teorías, favorecerán una dinámica de doble vía entre la universidad y el resto de la sociedad que puede ser muy potente y resignificar más que nunca el objetivo de la extensión universitaria.

No es la intención de este texto describir todas las posibilidades que dan las tecnologías en la actualidad, sino aportar algunos pocos ejemplos que permitan transmitir la idea. Otras tecnologías como la impresión 3D, la tecnología blockchain y la robótica son también elementos que están a la mano para generar disrupciones en los modelos universitarios.

Por último, parece también prudente cerrar esta sección mencionando algunas alertas que deben ser consideradas:

\section{Alerta 1}

Las instituciones que, por seguir la tendencia, implementen soluciones tecnológicas intentando reproducir modelos sin buscar sus respectivos roles en este nuevo ecosistema, tienen muchas posibilidades de fracasar. Cabe destacar, como un buen ejemplo, el Horizon Report para educación superior, que realizaba NMC (New Media Consortium's (NMC) y que fue adquirido en 2018 por EDUCAUSE. Su última edición EDUCAUSE Horizon Report 2019 Higher Education Edition (Alexander et al., 2019), es una excelente referencia para entender tendencias sobre aplicación de tecnologías en la educación superior (se describen tecnologías con sus respectivas estimaciones temporales de adopción, desafíos y problemas a resolver para su implantación, etc. Su revisión es muy importante, como fuente de inspiración y reflexión, pero de ninguna manera puede ser la única guía para la planificación y definición de la estrategia.

Hemos escuchado algunas universidades mencionar que ahora su objetivo debe ser enfocarse en la "experiencia del usuario", siguiendo la tan nombrada satisfacción del "cliente" (estudiante) que en tiempos de transformación digital se ha resignificado. Y las vemos incorporar tecnologías que posibiliten mejorar su satisfacción con algunos servicios que provee la universidad. No podemos asegurar que este pueda ser un objetivo a perseguir en sí mismo por parte de las universidades, lo cual no quiere decir que no importe. Estamos convencidos de que debe procurarse mejorar la experiencia de los usuarios (estudiantes y docentes). El punto es que no creemos que ese debiera ser el foco. Si el estudiante está en un lugar muy agradable, que lo hace feliz estar allí, pero ello no lo hace aprender mejor, no lo hace crecer intelectualmente, ¿esa universidad está cumpliendo su misión? Una institución que le provee todas las tecnologías disponibles para que su estadía en la universidad sea más fácil ¡está trabajando adecuadamente las habilidades de supervivencia y adaptabilidad que necesitan los graduados de hoy en día?

No estamos seguros de las respuestas, pero sí de que una universidad no debiera avanzar sin antes intentar contestarlas. Las universidades necesitan tener objetivos que le den un sentido dentro de la sociedad en la que se desempeñan, y son los que deben guiar su accionar. Si ello está claro, la tecnología puede ayudar, y mucho, a enfrentar los retos de la época para conseguirlos.

\section{Alerta 2}

La universidad podrá tomar un enfoque conservador, esperando que otras implementen primero las nuevas soluciones tecnológicas (y solo implementando lo ya probado); o tomar un enfoque proactivo e innovador, explorando alternativas nuevas. Pero en cualquiera de los casos necesita evaluar, reflexionar y tomar decisiones sobre cómo incorporará las tecnologías digitales en la universidad. No hacer nada, no es un enfoque conservador, es la opción más riesgosa que pone en peligro la propia institución.

En la actualidad, las decisiones sobre las tecnologías digitales en las universidades deben ser abordadas como un tema de alto impacto institucional, que no puede analizarse en forma disociada de la estrategia. Como hemos desarrollado previamente, las elecciones que se realicen sobre las TIC son determinantes en el futuro de la universidad y, por tanto, deben estar integradas en los procesos decisorios de la política universitaria. Habilitan y cierran posibilidades 
determinando su destino. Las definiciones estratégicas que tome la institución deben incluir las alternativas que posibilitan las nuevas tecnologías, y por otro lado, las definiciones sobre las tecnologías deben tomar como base las prioridades estratégicas de la universidad. Para que esto pueda suceder, debe existir en la institución una dinámica en donde las autoridades políticas estén al frente de la gobernanza de estas temáticas, y puedan participar activamente. Se precisa también un cargo de alto nivel, como responsable máximo de las TIC, que pueda compartir esa mesa de discusión, y luego ser el encargado de implementar estrategias y políticas definidas.

Parte de esas decisiones, es necesario revisar cuál es el presupuesto que la institución está dispuesta a invertir en las tecnologías. Si en los últimos diez años el porcentaje del presupuesto que se destinaba a este rubro no ha aumentado significativamente, cuando hemos ingresado en plena era digital, es un indicador de alerta que debiera observarse. No invertir lo suficiente tiene relación directa con las capacidades que tendrá esa institución para construir su futuro.

\section{Las instituciones de educación superior en la era digital: un rol clave para una sociedad del conocimiento más saludable}

Esta era que asombra y sorprende día a día con soluciones innovadoras también genera incomodidades y temores. Por un lado, ciertos hechos dan evidencia de una sociedad fácilmente manipulable, por el otro, la automatización de las elecciones basadas en las correlaciones y la concentración de estas herramientas en pocas manos.

Existen empresas que han logrado una concentración de datos y de herramientas de uso masivo que les permite la posibilidad de manipular los comportamientos de grandes porciones de la sociedad, con efectos económicos y políticos muy contundentes. Preocupa, por lo tanto, el almacenamiento masivo y concentrado de datos y del uso interesado que determinados actores puedan hacer de ellos.

También se proyecta un futuro donde modelos informatizados, basados en correlaciones de datos, serían los responsables de muchas de las elecciones que tomarían o no las personas (cuyas decisiones serían influenciadas). Estas correlaciones de datos tienen la característica de optimizar el orden establecido (porque se basan en el registro de lo sucedido en el pasado), sin embargo, existen muchos temas donde pareciera que este mundo necesita decisiones transformadoras de la realidad existente y no que se optimice (Chandler, 2015).

Las universidades pueden, y deseamos profundamente que lo hagan, jugar un rol que salvaguarde los objetivos de una sociedad más justa e igualitaria, elaborando teorías transformadoras de la realidad. Son instituciones que pueden pensar y ayudar a construir modelos superadores. Sus investigadores, docentes y estudiantes de múltiples disciplinas, aprovechando todo el potencial de los datos y las tecnologías existentes, deben trabajar hipótesis disruptivas que lo posibiliten. Además, pueden generar un canal de comunicación con la sociedad, que le permita a esta tener un actor independiente en la gestión del conocimiento. Es llevar el objetivo de extensión o vinculación con la sociedad de las universidades a su máxima relevancia, pero logrando sinergias con la investigación y la enseñanza y usando los mecanismos propios de la era digital.

Pueden ser, de algún modo, un contrapunto a ciertas corporaciones empresariales muy potentes en el dominio de la gestión del conocimiento en la era digital, y asumir un rol clave en la construcción de esta sociedad del conocimiento. Este no es un discurso anti capitalista o anti sistema, es simplemente uno que entiende a las universidades como los anticuerpos necesarios de la sociedad, que ayudarán a lograr un sistema más saludable.

Para lograr este objetivo, las universidades deben trabajar en forma asociativa, ya que el desafío es muy ambicioso, y también deben entender este aspecto como parte de su misión en esta nueva era.

\section{La OUI apoyando la agenda}

La Organización Universitaria Interamericana (OUI - www.oui-iohe.org), fundada en Canadá en 1980 y con su Secretariado en la ciudad de Montreal, es la única organización de educación superior 
presente de manera activa a nivel interamericano. Desde su misión resalta la cooperación de sus miembros y desde sus ejes estratégicos se articulan programas y actividades en apoyo a las instituciones miembros, fomentando la participación y el compromiso de apoyo mutuo entre las mismas. Compuesta por más de 350 miembros, entre instituciones de educación superior y asociaciones, que abarcan 28 países de las Américas.

La OUI apoya la innovación y cooperación en educación superior en las Américas. Cuenta con cinco programas (IGLU, COLAM, CAMPUS, EMULIES y EIESTEC) con un alto reconocimiento y calidad. Si bien difieren en su naturaleza y función, todos proporcionan capacitación a actores claves para que puedan entender mejor las prácticas adecuadas en materia de estrategias y las tendencias actuales que enfrentan, contribuyendo a su vez con la creación de espacios comunes de educación superior.

De manera más específica, los análisis realizados en el seno de la OUI respaldan la demostración de una diferencia entre las necesidades de cambios incurridos por la revolución digital descritas en este artículo y las prácticas actuales de gestión en las altas instancias de sus universidades miembros y en el medio de las diferentes unidades de gestión académicas y administrativas. Diferentes esfuerzos han sido realizados con el fin de reducir esta diferencia, específicamente por la presencia de "mesas redondas" de rectores en las conferencias de expertos en tecnología de la información (TI), cruzadas con paneles de expertos en TI dirigidos a rectores en sus reuniones, permitiendo de esta manera un mejor diálogo entre gestores y expertos. Sobre la base de estos intercambios sostenidos en el curso de los últimos años, las necesidades de formación especializada son evidentes y la OUI ha adoptado un eje referido a la innovación en su Plan Estratégico 2017-2022. Asimismo, diferentes iniciativas de formación y de capacitación han sido creadas en los diversos programas de la OUI.

En primer lugar, el Colegio de las Américas (COLAM), que ofrece una amplia gama de oportunidades de formación para profesionales universitarios y gubernamentales a través de Redes Interamericanas de expertos con sede en la Universidad Cooperativa de Colombia (UCC) en Medellín, inició el Programa Interamericano de Formación en Gestión de Ambientes de Innovación (PIFGAI). El objetivo del PIFGAI es desarrollar habilidades y competencias para impulsar y gestionar la puesta en marcha de ecosistemas de innovación viables y eficientes permitiendo un desempeño más eficaz de la labor de los gestores de ambientes de innovación. De manera específica, el programa busca que los participantes desarrollen habilidades y competencias que les permitan abordar las dimensiones relevantes que afectan el desarrollo organizacional y de procesos de ambientes de innovación, preparándolos a enfrentar los retos y las alertas planteados en este artículo.

En segundo lugar, el Instituto de Gestión y Liderazgo Universitario (IGLU), que forma nuevas generaciones de líderes universitarios desde 1983, está actualmente explorando la oportunidad para incorporar módulos de capacitación en transformación y estrategia digital de las universidades, buscando mejorar la comprensión y aplicación de conceptos y prácticas de gestión que posibiliten un uso más estratégico de las tecnologías y un enfoque que responda a los retos de la revolución digital, de tal manera que los participantes puedan estar en capacidad de identificar los requerimientos, participar activamente en el diseño de la transformación y la implementación de estrategias digitales, así como posibilitar su participación en la gobernanza de las tecnologías y los datos dentro de las universidades.

En tercer lugar, nació en el programa CAMPUS la iniciativa del espacio de movilidad virtual de educación superior (eMOVIES), para responder a las nuevas tendencias de la educación superior y al constante aumento de cursos virtuales, tanto en la calidad de los mismos como en el número de estudiantes que participan. En este contexto eMOVIES busca establecer las bases para la implementación de un Consorcio de Instituciones de Educación Superior (IES), que ejecute la movilidad de estudiantes en la modalidad educativa virtual, sobre la base de la reciprocidad académica y de acuerdo a los procedimientos y requisitos académicos establecidos por las "IES". Dicho movimiento pretende contribuir no solo a la oferta de una experiencia diversa y de calidad a sus estudiantes, 
sino también al reconocimiento de facto de la omnipresencia de los cursos en línea y la educación virtual como una oferta educativa que debe estandarizarse y fomentar sus procesos de reconocimiento académico.

Para concluir, es evidente que este tipo de iniciativas están siendo llamadas a aumentar en el seno de nuestra organización, así como en nuestros asociados en todo el continente. Otro ejemplo que da cuenta de la importancia de la temática para la OUI, es la colaboración en la publicación del libro "Cómo priorizas los proyectos TI estratégicos para tu universidad" (Fernández, Llorens, Juiz \& otros, 2018) que desarrolla un enfoque de gestión y gobernanza de las TI y que constituye una herramienta útil y vigente para la toma de decisiones en las IES.

\section{Conclusiones}

Tal como se ha planteado en las secciones previas, estamos transitando una nueva era: la denominada era digital. En primer término hemos evidenciado las principales tecnologías disruptivas que están generando un fuerte impacto en la sociedad. Son ejemplos la inteligencia artificial, Internet de las cosas, Blockchain, la analítica de datos, la realidad aumentada, etc. Luego se vio cómo la apropiación de estas tecnologías por las organizaciones y las personas está transformando los modelos y procesos, generando cambios profundos, abruptos y a la vez efímeros.

Desde dicho lugar se analizaron los riesgos y oportunidades para las universidades. En ese marco, también se plateó que la transformación digital de las instituciones de educación superior es una necesidad, que debe ser abordada sin dilaciones, pero también con un enfoque crítico y reflexivo bajo las particularidades de cada institución.

Por último, también se ha intentado fijar una posición sobre el rol que las instituciones de educación superior pueden asumir en esta era: ser generadoras y difusoras de conocimiento, y contribuir a la construcción de una sociedad del conocimiento más saludable, generando teorías transformadoras de la realidad, no necesariamente basadas en la optimización del orden preexistente. Por otro lado, que se conviertan en una canal de distribución del conocimiento confiable para la sociedad, entendiendo que estas instituciones no tiene otro fin que la generación y distribución de conocimiento sin segundas intenciones.

En conclusión, el objetivo de este artículo es generar la semilla para habilitar espacios de discusión, de capacitación y motivar los planes que recorran este camino; donde también se propone a la Organización Universitaria Interamericana (OUI) como un espacio propicio para abrir el debate en relación con la transformación que necesitan las universidades en la nueva era digital.

Esperamos haber contribuido a circunscribir mejor los principales desafíos en torno a esta temática y reiteramos nuestro compromiso de continuar innovando con la propuesta de alternativas viables en el plan de la cooperación y de la formación, con el fin de reducir las diferencias de la brecha digital.

\section{Referencias}

Alexander, B., Ashford-Rowe, K., Barajas-Murphy, N., Dobbin, G., Knott, J., McCormack, M., ... Weber, N. (2019). EDUCAUSE Horizon Report: 2019 Higher Education Edition. 44.

Ariño, M. (2018). Transformación digital: Puntos de interés y consideraciones para la reflexión en la transformación digital en la universidad. RUIDERAe: Revista de Unidades de Información., 13. Recuperado de https://revista.uclm.es/index. php/ruiderae/article/view/1833/143.

Bostrom, N. (2014). Superintelligence: paths, dangers, strategies. Oxford, Reino Unido: Oxford University Press.

Chandler, D. (2015). A world without causation: Big data and the coming of age of posthumanism. Millennium-Journal of International Studies, 43(3), 833-851.

Chinkes, E., Fernandez Blanco, M. L., \& Coronel, L. (septiembre 2015). BIG DATA: El Dato en un Rol Estratégico, un Desafío para las Soluciones de Gestión de Datos. Presentado en Jornada Académica del Departamento Pedagógico de Sistemas 2015, en la Facultad de Ciencias Económicas de la Universidad de Buenos Aires. Buenos Aires, Argentina. 
Davenport, T. H., \& Westerman, G. (8 de septiembre 2019). ¿Por qué fracasaron las transformaciones digitales de General Electric y Nike? Harvard Business Review en español. Recuperado de https:// hbr.es/modelos-de-negocio/1309/por-qu-fracasaron-las-transformaciones-digitales-de-general-electric-y-nike.

3Dnative. El sitio web de la impresión 3D. (s. f.). Recuperado de https://www.3dnatives.com/es/

Domo. (2018). Data Never Sleeps 6. Recuperado de https://www.domo.com/learn/data-never-sleeps-6.

Fernández, A., Llorens F. Juiz C. \& otros (2018), Cómo priorizar los proyectos TI estratégicos para tu universidad, Alicante, España: Universidad de Alicante. Recuperado de: http://rua.ua.es/ dspace/handle/10045/86867.
Grech, A., \& Camilleri, A. F. (2017). Blockchain in Education. EUR.

Hildebrand, C. (2014). Ray Wang: CloudIs The "Foundation For Digital Transformation» Recuperado de https:// www.forbes.com/sites/oracle/2014/12/19/ ray-wang-cloud-is-the-foundation-for-digital-transformation/.

Kitchin, R. (2014). Big Data, new epistemologies and paradigm shifts. Big Data \& Society, 1(1), doi: 205395171452848. doi. org/10.1177/2053951714528481.

Schwab, K. (2016). La cuarta revolución industrial. Debate.Recuperadodehttps://books.google.com/ books/about/La_cuarta_revoluci\%C3\%B3n_industrial.html?hl=es\&id=BRonDQAAQBAJ 